\title{
An electronic audit tool to track the donning and doffing practices of personal protective equipment in the emergency department.
}

\section{Maya Jusza, Ramya Sridhar, Jennifer Woods, Sharon Drury}

Background: Maintaining the safety of patients and healthcare professionals is a priority in all healthcare settings. Infection prevention measures such as donning and doffing practices of personal protective equipment (PPE) have become even more imperative in light of the SARSCoV-2 pandemic. Potential PPE breaches and the degree of frontline compliance are currently being analyzed through the use of paper PPE audit tools which can be laborious and timeconsuming. The development of an electronic alternative would improve frontline safety and enhance the efficiency of data collection, while optimizing the ability to share these observations with the frontline team in real time. Two nursing leadership students from the University of Alberta were tasked with developing an electronic PPE audit tool prototype for the University of Alberta Hospital's emergency department.

Implementation: An electronic PPE audit tool prototype was developed using Google Forms which provided a user-friendly interface. Google applications were used as no confidential or patient data was collected during PPE audits. The prototype auto-populated the data entries into linked spreadsheets and interactive data dashboards that visualized the data using graphs in real time. This enabled users to easily identify trends and direct educational interventions as required. Instructional one pagers and screencast videos were also created to accompany the prototype.

The prototype was reviewed by and received extensive support from: Unit Managers, Patient Care Managers, Process Improvement Nurses, Infection Prevention Control (IPC), the Executive Director of the University of Alberta Hospital and Stollery emergency department and Edmonton Zone medicine programs, and the University of Alberta Hospital and Mazankowski Executive Leadership Team. Several changes and improvements were made using the Plan-Do-Study-Act cycle. This prototype has currently been replicated onto an Alberta Health Services (AHS) server and has completed the formal testing phase with a planned application launch date.

Evaluation Methods: Plan-Do-Study-Act cycles were used to guide the implementation of this audit tool prototype. After development, the prototype was tested and revised which included six rounds of audit trials at the University of Alberta Hospital's emergency department and on some inpatient medicine units. This prototype was consistently evaluated at various stages of development and changes were made to include feedback. After approval was received to recreate this prototype onto an AHS compatible server, additional changes were made to ensure functionality. These changes included adding designations and simplifying certain questions. IPC was consulted to ensure the steps outlined for donning and doffing in the prototype were accurate and reflected requirements in the clinical environment.

Results: This audit tool prototype has gathered tremendous support through various demonstrations of its ability to streamline data collection in the healthcare setting. This data is 
relevant to the safety of both frontline workers and patients as it identifies inconsistencies in donning and doffing practices. In addition, the prototype also complements the Edmonton zonewide PPE coaching initiative by allowing for a quantitative measurement of its efficacy. This has prompted the fast-tracked replication of an AHS compatible version with the assistance of a dedicated team that includes the creators of the prototype tool, IPC, Quality Assurance, Information Technology, and Clinical Services Development. This version has a scheduled launch date on March 22, 2021 and is to be initially rolled out to University of Alberta's emergency department and medicine units. The objective is to eventually make this the standardized PPE audit tool throughout Alberta.

\section{Advice and Lessons Learned:}

1) In order to be sustainable and implemented site-wide, an AHS compatible tool is required. The use of Google applications is not preferred as data will be stored outside of the AHS server. Even though there is no confidential information, wide-spread use may overwhelm the Google platform and a Gmail account is required to view data. An inhouse AHS alternative has been developed.

2) Several discussions took place regarding discretions on what steps can be audited according to IPC protocols. For example, some clinical nurse educators prefer hand hygiene to be completed between donning steps, while this is not mandatory or auditable according to IPC. In addition, several discussions took place to identify the operational and business owners of this tool which are required to support the AHS compatible version of the application.

3) PPE audits are vital across all hospital departments to improve the quality of healthcare. The use of PPE during patient care has grown exponentially due to the SARS-CoV2 pandemic and has amplified the need for an electronic alternative to the existing paper PPE audit tool. The electronic audit tool offers an innovative way to accurately and efficiently collect and display data which will promote an improved quality of care. 